DESY 01-112

hep-ph/0108110

August 2001

\title{
Heavy-Light Meson Decay Constant from QCD Sum Rules in Three-Loop Approximation
}

\author{
A.A. Penin $\stackrel{\ddagger}{\ddagger}$ and M. Steinhauser \\ II. Institut für Theoretische Physik, \\ Universität Hamburg, D-22761 Hamburg, Germany
}

\begin{abstract}
In this paper we compute the decay constant of the pseudo-scalar heavy-light mesons in the heavy quark effective theory framework of QCD sum rules. In our analysis we include the recently evaluated three-loop result of order $\alpha_{s}^{2}$ for the heavy-light current correlator. The value of the bottom quark mass, which essentially limits the accuracy of the sum rules for $B$ meson, is extracted from the nonrelativistic sum rules for $\Upsilon$ resonances in the next-to-next-to-leading approximation. We find stability of our result with respect to all types of corrections and the specific form of the sum rule which reduces the uncertainty. Our results $f_{B}=206 \pm 20 \mathrm{MeV}$ and $f_{D}=195 \pm 20 \mathrm{MeV}$ for the $B$ and $D$ meson decay constants are in impressive agreement with recent lattice calculations.
\end{abstract}

PACS numbers: 12.38.Lg, 14.40.Nd, 14.40.Lb

\section{Introduction}

The decay constant of a pseudo-scalar meson with one heavy and one light quark constitutes a hadronic quantity which is of primary phenomenological interest. It covers the strength of the leptonic weak decays of $B$ and $D$ mesons and enters as an input quantity into the analysis of the nonleptonic $B$ and $D$ meson decays and the $B-\bar{B}$ mixing process. The latter is of special interest since it provides a direct source of the information on the Cabibbo-Kobayashi-Maskawa matrix elements involving the top quark. Still no experimental information of sufficient accuracy is available and the theoretical study of the decay constant is mandatory.

The first quantitative evaluation of the $B$ and $D$ meson decay constants $f_{B}$ and $f_{D}$ using radiative corrections was performed in [1] where the QCD sum rules proposed

\footnotetext{
${ }^{\ddagger}$ Permanent address: Institute for Nuclear Research, Russian Academy of Sciences, 60th October Anniversary Prospect 7a, Moscow 117312, Russia.
} 
in [2, 3] have been used together with the perturbative two-loop results of $\mathcal{O}\left(\alpha_{s}\right)$ [4]. More refined evaluations followed [5, 6, 0, 8, 9] where it was realized that the accuracy of the decay constants is significantly limited by the uncertainty of the bottom quark mass. In [10, 11, 12 the heavy quark effective theory (HQET) 13, 14 has been used to resum the leading and next-to-leading logarithms of the heavy quark mass. After the renormalization group improvement the two-loop corrections were found to be huge which might be a signal of the importance of the higher order contributions and questions the reliable determination of the decay constants.

The only method to compute hadronic matrix elements which is entirely based on the first principles of QCD are probably the lattice gauge theory simulations. For the $B$ meson decay constant the value

$$
f_{B}^{\text {lat }}=200 \pm 30 \mathrm{MeV},
$$

is cited in the review article [15] as an average over different calculations performed on the lattice. It agrees well with a recent evaluation of $f_{B}$ [16] where the result $f_{B}=$ $204(8)(29)(+44) \mathrm{MeV}$ is given. Here, the first error is statistical, the second due to the discretization and the third one includes the uncertainty from the lattice scale. The numbers obtained for $f_{B}$ from lattice calculations are in reasonable agreement with the ones obtained from sum rules. An averaged value for the latter can be found in [17]

$$
f_{B}^{\text {s.r. }}=178 \pm 42 \mathrm{MeV} .
$$

For the $D$ meson decay constant the situation is similar. The lattice result [15, 18]

$$
f_{D}^{\text {lat }}=225 \pm 30 \mathrm{MeV},
$$

again overshoots the sum-rule estimate

$$
f_{D}^{\text {s.r. }}=188 \pm 48 \mathrm{MeV},
$$

given in Ref. [17]. In spite of the essential progress in the lattice calculations their uncertainty is still rather large and it is too early to rely solely on the results of this approach. On the other hand, the sum-rule analysis can be essentially improved to reach an accuracy which is comparable or even better than the current accuracy of the lattice calculations and provides an independent cross check of the latter. In this paper we perform the analysis of the sum rules for the pseudo-scalar heavy-light meson decay constant and improve the existing calculations with respect to several points which we summarize in the following:

(i) We include the three-loop perturbative corrections of $\mathcal{O}\left(\alpha_{s}^{2}\right)$ which recently became available [19, 20].

(ii) The bottom quark mass, which constitutes a crucial input for $f_{B}$, is determined using the approach suggested in [21, 22] in the context of semileptonic decays of the bottom quark. The latter is also quite sensitive to the pole mass $m_{b}$. The basic idea is to replace $m_{b}$ by the ratio of experimental and theoretical moments of the nonrelativistic sum rules 
for $\Upsilon$ resonances. It is computed order by order up to the next-to-next-to-leading accuracy depending on the perturbative input used for the evaluation of the sum rules for $f_{B}$. In this context we would like to refer to [7] where also the $\Upsilon$-resonance sum rules have been used, however, only up to order $\alpha_{s}$.

The charm quark pole mass $m_{c}$, necessary for the calculation of $f_{D}$, is then extracted from the HQET relation between $m_{b}$ and $m_{c}$.

(iii) For the evaluation of the decay constant $f_{B}$ we use both Laplace and Hilbert sum rules. We present, for the first time, the explicit formulae of the latter in the framework of HQET. The comparison of the results obtained with both approaches provides us with an estimate of the intrinsic uncertainty of the method.

We will show that these new ingredients improve significantly the reliability of the sum rules and finally the prediction for the decay constant, in particular for $f_{B}$.

This paper is organized as follows. In Sections 2 and 3 we introduce the basic features of the sum rules and the HQET formalism, respectively. In Section 1 the renormalization group improved sum rules for the decay constant are given in the three-loop approximation. The problem of the heavy quark mass determination is discussed in Section 5. In Section 6, we present the numerical analysis and Section 0 contains our conclusions.

\section{Sum rules}

The decay constant of a pseudo-scalar meson $P$ consisting of a heavy $(Q)$ and a light quark $(q)$ is defined through the matrix element

$$
\left\langle 0\left|j_{\mu}^{a}\right| P(p)\right\rangle=i f_{P} p_{\mu},
$$

where $j_{\mu}^{a}=\bar{q} \gamma_{\mu} \gamma_{5} Q$ is the axial-vector current. In this paper we focus on the $B$ meson with the bottom quark as the heavy constituent but present also the analysis of $D$ mesons with charm as heavy flavour. We neglect the $S U(3)$ violating effects of the strange quark mass. The ratio of the decay constants of strange and non-strange mesons can be reliably computed both on the lattice and using QCD sum rules as an essential part of the uncertainties cancel [17, 15, 16, 18].

In order to derive the QCD sum rules for the pseudo-scalar heavy-light meson decay constant $f_{P}$ one considers the correlator

$$
\Psi^{a}\left(q^{2}\right)=i \int \mathrm{d} x e^{i q x}\left\langle 0\left|T \partial^{\mu} j_{\mu}^{a}(x) \partial^{\nu} j_{\nu}^{a \dagger}(0)\right| 0\right\rangle,
$$

which is related to the correlator of the pseudo-scalar currents

$$
\Pi^{p}\left(q^{2}\right)=i \int \mathrm{d} x e^{i q x}\left\langle 0\left|T j^{p}(x) j^{p \dagger}(0)\right| 0\right\rangle
$$

by the equation $\partial^{\mu} j_{\mu}^{a}=m_{Q} j^{p}$, where $j^{p}=i\left(m_{Q}(\mu) / m_{Q}\right) \bar{q} \gamma^{5} Q$ and $\bar{m}_{Q}(\mu)$ and $m_{Q}$ are the $\overline{\mathrm{MS}}$ and pole mass of the heavy quark $Q$, respectively.

Following [3] the correlator $\Psi^{a}\left(q^{2}\right)$ is evaluated in two ways. In the Euclidean region where $q^{2}-m_{Q}^{2} \ll 0$ it can be reliably computed in QCD because of the asymptotic 
freedom. The correlator gets a perturbative contribution corresponding to the leading operator in the operator product expansion (OPE) of the two currents in Eq. (6). Furthermore there are power suppressed terms from the vacuum expectation values of the higher dimension operators (so-called vacuum condensates) parameterizing the nonperturbative long-distance effects. On the other hand, the correlator can be obtained from the dispersion integral over the physical states. In the standard analysis only the ground state meson is supposed to give a delta-function contribution to the spectral function. Assuming local quark-hadron duality the contribution from the higher resonances is modeled by the perturbative continuum starting at some threshold $s_{c}$ which brings an intrinsic uncertainty to the approach. Finally one arrives at the following equation

$$
\Psi_{\mathrm{pt}}^{a}\left(q^{2}\right)+\Psi_{\mathrm{npt}}^{a}\left(q^{2}\right)=\frac{f_{P}^{2} M_{P}^{4}}{M_{P}^{2}-q^{2}}+\frac{1}{\pi} \int_{s_{c}}^{\infty} \mathrm{d} s \frac{\operatorname{Im}\left[\Psi_{\mathrm{pt}}^{a}(s)\right]}{s-q^{2}}+\text { subtractions },
$$

where $M_{P}$ is the meson mass. $\Psi_{\mathrm{pt}}^{a}$ and $\Psi_{\mathrm{npt}}^{a}$ are the perturbative and nonperturbative QCD contributions, respectively. The subtractions needed for the dispersion integral are not specified explicitly as they will drop out in the following. To suppress the contribution from higher resonances and to reduce the uncertainty one can perform a Borel transformation of Eq. (8)

$$
\left.\frac{1}{(n-1) !}\left(-q^{2} \frac{\mathrm{d}}{\mathrm{d} q^{2}}\right)^{n} \Psi^{a}\left(q^{2}\right)\right|_{\substack{-q^{2}, n \rightarrow \infty \\-q^{2} / n=M^{2}}}=\frac{1}{\pi} \int_{m_{Q}^{2}}^{\infty} \mathrm{d} s \frac{e^{-s / M^{2}}}{M^{2}} \operatorname{Im}\left[\Psi^{a}(s)\right],
$$

where $M$ is the Borel parameter, and arrive at so-called Laplace sum rules. Alternatively, it is possible to consider moments of Eq. (8)

$$
\left.\frac{1}{n !}\left(\frac{\mathrm{d}}{\mathrm{d} q^{2}}\right)^{n} \Psi^{a}\left(q^{2}\right)\right|_{q^{2}=0}=\frac{1}{\pi} \int_{m_{Q}^{2}}^{\infty} \mathrm{d} s \frac{\operatorname{Im}\left[\Psi^{a}(s)\right]}{s^{n+1}},
$$

which leads to Hilbert sum rules. To estimate the intrinsic uncertainty of the method we will follow both options. In the above sum rules the weight functions cut off the dispersion integral at a typical hadronic scale much less than the heavy quark mass so that it is saturated by the near threshold region where the heavy quark is nonrelativistic. Laplace sum rules are particularly relevant for the nonrelativistic HQET analysis because of the heavy quark mass independent exponential suppression of the relativistic momentum region. The situation is more tricky in the case of Hilbert sum rules as is discussed below.

\section{$3 \quad$ Heavy quark effective theory}

Systematic description of the heavy quark nonrelativistic dynamics and consistent separation of the relativistic effects can be done within HQET. Let us discuss this issue in more detail. The perturbative heavy-light quark system involves two dynamical scales: 
the hard scale given by the heavy quark mass and the soft scale given by the off-threshold energy

$$
\tilde{\omega}=\frac{q^{2}-m_{Q}^{2}}{m_{Q}} .
$$

By integrating out the relativistic hard modes with the off-shell momentum of order $m_{Q}$ one arrives at HQET which includes $\tilde{\omega}$ as dynamical scale. The effect of the hard modes is accumulated in the Wilson (matching) coefficients leading to an expansion in $\alpha_{s}$ along with relativistic corrections and contributions from higher dimensional operators leading to an expansion in $1 / m_{Q}$. In the hadronic matrix elements the latter is converted to an expansion in the dimensionless parameter $\bar{\Lambda} / m_{Q}$ where $\bar{\Lambda} \approx M_{P}-m_{Q}$ describes the nonperturbative long-distance effects and remains finite as $m_{Q} \rightarrow \infty$. In the process of scale separation spurious divergences appear at the intermediate steps which result in the anomalous dimensions of the effective theory operators and lead to the corrections involving the large logarithms of the form $\ln \left(m_{Q} / \tilde{\omega}\right)$. These logarithmic corrections can be resummed by solving the effective theory renormalization group equations.

For this purpose let us consider the effective theory realization of the axial-vector current. The corresponding connection between the QCD operator and its HQET counterpart is given by

$$
j_{\mu}^{a}=C_{a}\left(m_{Q}\right) \tilde{C}\left(m_{Q}\right) \tilde{j}_{\mu}^{\prime}\left(m_{Q}\right)+\mathcal{O}\left(1 / m_{Q}\right) .
$$

The matching coefficients have been computed in [23, 24] up to order $\alpha_{s}^{2}$. In the $\overline{\mathrm{MS}}$ subtraction scheme they read

$$
\begin{aligned}
C_{a}\left(M_{Q}\right)= & 1-\frac{\alpha_{s}^{\left(n_{f}\right)}\left(m_{Q}\right)}{\pi} \frac{2}{3}+\left(\frac{\alpha_{s}^{\left(n_{f}\right)}\left(m_{Q}\right)}{\pi}\right)^{2}\left[-\frac{683}{576}-\frac{17 \pi^{2}}{72}-\frac{\pi^{2}}{18} \ln 2-\frac{11}{36} \zeta(3)\right. \\
& \left.+n_{l}\left(\frac{47}{288}+\frac{\pi^{2}}{36}\right)\right] \\
\tilde{C}\left(m_{Q}\right)= & 1+\frac{89}{864}\left(\frac{\alpha_{s}^{\left(n_{l}\right)}\left(m_{Q}\right)}{\pi}\right)^{2}
\end{aligned}
$$

where $n_{l}$ is the number of light flavours and $\zeta(3)=1.2020569 \ldots$ is the Riemann $\zeta$ function. The renormalization group equation which governs the evolution of $\tilde{j}_{\mu}^{\prime}(\mu)$ is of the form

$$
\mu^{2} \frac{\mathrm{d}}{\mathrm{d} \mu^{2}} \tilde{j}_{\mu}^{\prime}(\mu)=\tilde{\gamma}^{\prime} \tilde{j}_{\mu}^{\prime}(\mu)
$$

where the anomalous dimension is known up to two loops 25

$$
\begin{aligned}
& \tilde{\gamma}^{\prime}=\gamma_{0} \frac{\alpha_{s}^{\left(n_{l}\right)}}{\pi}+\gamma_{1}\left(\frac{\alpha_{s}^{\left(n_{l}\right)}}{\pi}\right)^{2}+\mathcal{O}\left(\alpha_{s}^{3}\right), \\
& \gamma_{0}=\frac{1}{2}, \quad \gamma_{1}=\frac{127}{144}+\frac{7 \pi^{2}}{108}-\frac{5}{72} n_{l} .
\end{aligned}
$$


The solution of Eq. (14) reads

$$
\tilde{j}_{\mu}^{\prime}(\mu)=\sqrt{\frac{X\left(m_{Q}\right)}{X(\mu)}} \tilde{j}_{\mu}^{\prime}\left(m_{Q}\right)
$$

where

$$
X(\mu)=\left(\alpha_{s}^{\left(n_{l}\right)}(\mu)\right)^{2 \gamma_{0} / \beta_{0}}\left[1+\left(\frac{\gamma_{1}}{\beta_{0}}-\frac{\gamma_{0} \beta_{1}}{\beta_{0}^{2}}\right) \frac{\alpha_{s}^{\left(n_{l}\right)}(\mu)}{\pi}+\mathcal{O}\left(\alpha_{s}^{2}\right)\right]^{2},
$$

and the first two coefficients of the $\beta$-function are given by

$$
\beta_{0}=\frac{11}{4}-\frac{1}{6} n_{l}, \quad \beta_{1}=\frac{51}{8}-\frac{19}{24} n_{l} .
$$

Thus in the nonrelativistic region $\tilde{\omega} \ll m_{Q}$ we have the following representation of the perturbative part of the correlator (6)

$$
\operatorname{Im}\left[\Psi_{\mathrm{pt}}^{a}\left(q^{2}\right)\right]=\left(C_{a}\left(m_{Q}\right) \tilde{C}\left(m_{Q}\right)\right)^{2} \frac{X(\mu)}{X\left(m_{Q}\right)} m_{Q}^{2} \operatorname{Im}\left[\tilde{\Pi}_{\mathrm{pt}}(\tilde{\omega})\right]+\mathcal{O}\left(1 / m_{Q}\right),
$$

where the universal HQET current correlator $\tilde{\Pi}_{\mathrm{pt}}(\tilde{\omega})$ does not depend on $m_{Q}$ and the spin and parity of the currents. Its imaginary part is known up to the three-loop approximation of $\mathcal{O}\left(\alpha_{s}^{2}\right)$ [2]]

$$
\begin{aligned}
\operatorname{Im}\left[\tilde{\Pi}_{\mathrm{pt}}(\tilde{\omega})\right]= & \frac{3 \tilde{\omega}^{2}}{8 \pi}\left\{1+\frac{\alpha_{s}^{\left(n_{l}\right)}(\mu)}{\pi}\left[\frac{17}{3}+\frac{4 \pi^{2}}{9}+L_{\tilde{\omega}}\right]+\left(\frac{\alpha_{s}^{\left(n_{l}\right)}(\mu)}{\pi}\right)^{2}[99(15)\right. \\
& +\left(\frac{1657}{72}+\frac{97 \pi^{2}}{54}\right) L_{\tilde{\omega}}+\frac{15}{8} L_{\tilde{\omega}}^{2}+n_{l}\left(-3.6(4)+\left(-\frac{13}{12}-\frac{2 \pi^{2}}{27}\right) L_{\tilde{\omega}}\right. \\
& \left.\left.\left.-\frac{1}{12} L_{\tilde{\omega}}^{2}\right)\right]\right\}
\end{aligned}
$$

where $L_{\tilde{\omega}}=\ln \left(\mu^{2} / \tilde{\omega}^{2}\right)$. The uncertainty in the non-logarithmic three-loop terms results from the semi-numerical method used in Ref. [20]. For $\mu \approx \tilde{\omega}$ the HQET correlator does not include large logarithms. They are all contained in the factor $X(\mu) / X\left(m_{Q}\right)$ which sums up the leading and next-to-leading logarithms of the form $\alpha_{s}^{n} \ln ^{n}\left(m_{Q} / \tilde{\omega}\right)$ and $\alpha_{s}^{n+1} \ln ^{n}\left(m_{Q} / \tilde{\omega}\right)$.

On the phenomenological side the HQET decay constant is defined through the matrix element of the HQET current

$$
\left\langle 0\left|\tilde{j}_{\mu}(\mu)\right| \tilde{P}(v)\right\rangle=\frac{i}{\sqrt{2}} \tilde{f}_{P}(\mu) v_{\mu},
$$

where $|\tilde{P}(v)\rangle$ is the nonrelativistic, i.e. quantum mechanical, meson state with velocity $v$. It is connected to $f_{P}$ via the relation

$$
f_{P} \sqrt{M_{P}}=C_{a}\left(m_{Q}\right) \tilde{C}\left(m_{Q}\right) \tilde{f}_{P}\left(m_{Q}\right)+\mathcal{O}\left(1 / m_{Q}\right) .
$$


By using the renormalization group property of the HQET current it is convenient to introduce the renormalization group invariant quantity

$$
\tilde{f}_{P}^{r}=\sqrt{X(\mu)} \tilde{f}_{P}(\mu)
$$

which is a universal low-energy parameter of strong interactions.

\section{The heavy quark effective theory sum rules}

Now we are in the position to write down the renormalization group improved sum rules for the heavy-light pseudo-scalar meson decay constant.

\subsection{Laplace sum rules}

Let us start with the sum rules in the infinite heavy quark mass limit $m_{Q} \rightarrow \infty$. Transforming Eq. (8) to HQET and neglecting the mass suppressed terms we obtain

$$
\left(\tilde{f}_{P}^{r}\right)^{2}=\mathrm{e}^{\Delta / T}\left\{X(\mu)\left[\frac{T}{\pi} \int_{0}^{\tilde{\omega}_{c} / T} \mathrm{~d} z e^{-z} \operatorname{Im}\left[\tilde{\Pi}_{\mathrm{pt}}(z T)\right]-\langle\bar{q} q\rangle(\mu)\left[1+2 \frac{\alpha_{s}}{\pi}\right]\right]+X(T) \frac{m_{0}^{2}\langle\bar{q} q\rangle}{4 T^{2}}\right\},
$$

where $\Delta=\left(M_{P}^{2}-m_{Q}^{2}\right) / m_{Q}, \tilde{\omega}_{c}=\left(s_{c}-m_{Q}^{2}\right) / m_{Q}, m_{0}^{2}=\left\langle\bar{q} g_{s} G^{\mu \nu} \sigma_{\mu \nu} q\right\rangle /\langle\bar{q} q\rangle$ and $m_{Q} T=$ $M^{2}$. We keep the operators up to dimension five in the OPE and neglect the running of the quark-gluon operator.

The mass suppressed contribution to the correlator can be found by subtracting the asymptotic HQET result from the full theory expression. In this way the mass suppressed contribution to the physical decay constant from Laplace sum rules is obtained as

$$
\begin{aligned}
\delta f_{P}^{2}= & \mathrm{e}^{\Delta / T} \frac{1}{M_{P}}\left(\frac{m_{Q}}{M_{P}}\right)^{3}\left\{\frac{T}{\pi} \int_{0}^{\tilde{\omega}_{c} / T} \mathrm{~d} z e^{-z} \operatorname{Im}\left[\delta \Pi_{\mathrm{pt}}^{p}\right](z T)\right. \\
& \left.+\langle\bar{q} q\rangle\left(m_{Q}\right)\left[\frac{2 \alpha_{s}}{\pi} \frac{T}{m_{Q}} \int_{0}^{\infty} \mathrm{d} z \frac{\mathrm{e}^{-z}}{1+z T / m_{Q}}\right]-\frac{m_{0}^{2}\langle\bar{q} q\rangle}{2 T m_{Q}}+\frac{\left\langle\alpha_{s} G^{\mu \nu} G_{\mu \nu}\right\rangle}{12 \pi m_{Q}}\right\},
\end{aligned}
$$

where

$$
\operatorname{Im}\left[\delta \Pi_{\mathrm{pt}}^{p}\left(q^{2}\right)\right]=\operatorname{Im}\left[\Pi_{\mathrm{pt}}^{p}\left(q^{2}\right)\right]-\left(C_{a}\left(m_{Q}\right) \tilde{C}\left(m_{Q}\right)\right)^{2} \frac{X(\mu)}{X\left(m_{Q}\right)} \operatorname{Im}\left[\tilde{\Pi}_{\mathrm{pt}}(\tilde{\omega})\right] .
$$

The one-loop expression for this function is given by

$$
\operatorname{Im}\left[\delta^{(1)} \Pi_{\mathrm{pt}}^{p}(\tilde{\omega})\right]=-\frac{3}{8 \pi} \frac{\tilde{\omega}}{m_{Q}} \frac{\tilde{\omega}^{2}}{1+\tilde{\omega} / m_{Q}} .
$$

The two-loop approximation for $\Pi_{\mathrm{pt}}^{p}\left(q^{2}\right)$ is known in full QCD in analytical form [4. It determines the $\mathcal{O}\left(\alpha_{s}\right)$ part of Eq. (26) which reads

$$
\operatorname{Im}\left[\delta^{(2)} \Pi_{\mathrm{pt}}^{p}(\tilde{\omega})\right]=\frac{\alpha_{s}}{2 \pi^{2}} \frac{\tilde{\omega}^{2}}{1+\tilde{\omega} / m_{Q}}\left[-\frac{\tilde{\omega}}{m_{Q}}\left(\frac{13}{4}+\frac{\pi^{2}}{3}+\frac{3}{2} \ln \left(\frac{m_{Q}}{\tilde{\omega}}\right)\right)+F\left(\frac{\tilde{\omega}}{m_{Q}}\right)\right]
$$


where

$$
\begin{aligned}
F(x) & =2 \operatorname{Li}_{2}(-x)+\ln (x) \ln (1+x)-\frac{x}{1+x} \ln (x)+\frac{1+x}{x} \ln (x+1)-1 \\
& =-\frac{3}{2} x+\left(\frac{1}{3}+\frac{1}{2} \ln (x)\right) x^{2}+\mathcal{O}\left(x^{3}\right)
\end{aligned}
$$

with $\mathrm{Li}_{2}(z)$ being the dilogarithmic function. The perturbative mass suppressed contribution of $\mathcal{O}\left(\alpha_{s}^{2}\right)$ can be obtained from the numerical three-loop result of [19, 20] which is available under the URL http://www-ttp.physik.uni-karlsruhe.de/Progdata/ ttp00-25. Thus we get the final expression for the decay constant

$$
f_{P}^{2}=\left(\frac{m_{Q}}{M_{P}}\right)^{3} \frac{\left(C_{a}\left(m_{Q}\right) \tilde{C}\left(m_{Q}\right)\right)^{2}}{X\left(m_{Q}\right)} \frac{\left(\tilde{f}_{P}^{r}\right)^{2}}{M_{P}}+\delta f_{P}^{2}
$$

The first term of this equation includes the leading HQET contribution up to $\mathcal{O}\left(\alpha_{s}^{2}\right)$ and the resummed leading and next-to-leading logarithms of the heavy quark mass. The second term represents all heavy quark mass suppressed terms up to order $\alpha_{s}^{2}$.

\subsection{Hilbert sum rules}

The use of HQET for Hilbert sum rules is rather subtle as they do not have a proper infinite heavy quark mass limit. Indeed, the naive limit $m_{Q} \rightarrow \infty$ leads to the decay constant

$$
\left(\tilde{f}_{P}^{r}\right)^{2}=\frac{1}{8 \pi^{2}} \tilde{\omega}_{c}^{3}-\langle\bar{q} q\rangle+\mathcal{O}\left(\alpha_{s}\right)
$$

which, in contrast to Eq. (24), does not contain a dynamical constraint on the parameter $\tilde{\omega}_{c}$. Therefore one cannot use them to study the HQET decay constant. Nevertheless it is possible to apply Hilbert sum rules for the calculation of the physical decay constant and, furthermore, use HQET for the analysis. Indeed, if we keep the factor $1 / s^{n+1}=$ $1 /\left(m_{Q}^{2 n+2}\left(1+\tilde{\omega} / m_{Q}\right)^{n+1}\right)$ unexpanded in $1 / m_{Q}$, it is straightforward to obtain the proper scaling

$$
\tilde{\omega}_{c}=\frac{4}{3} \Delta+\mathcal{O}\left(\alpha_{s}, 1 / m_{Q}\right)
$$

where $\Delta$ is defined after Eq. (24), from the ratio of two arbitrary moments. Note that Eq. (32) is obtained from the purely perturbative correlator and the quark condensate contribution is neglected. This allows for the nonrelativistic treatment of the heavy quark in $\Psi^{a}(s)$. Moreover, the dispersion integral is saturated by the region $\tilde{\omega}<m_{Q} / n$ and thus for $n>m_{Q} / \tilde{\omega}_{c}$ the result is not sensitive to $\tilde{\omega}_{c}$ in contrast to Eq. (31). In this way we obtain the renormalization group improved Hilbert sum rules of the following form

$$
f_{P}^{2}=\frac{M_{P}^{2 n-2}}{m_{Q}^{2 n-1}}\left\{\frac { \tilde { \omega } _ { c } } { \pi } \int _ { 0 } ^ { 1 } \frac { \mathrm { d } z } { ( 1 + z \tilde { \omega } _ { c } / m _ { Q } ) ^ { n + 1 } } \left(\left(C_{a}\left(m_{Q}\right) \tilde{C}\left(m_{Q}\right)\right)^{2} \frac{X(\mu)}{X\left(m_{Q}\right)} \operatorname{Im}\left[\tilde{\Pi}_{\mathrm{pt}}\left(z \tilde{\omega}_{c}\right)\right]+\right.\right.
$$




$$
\begin{aligned}
& \left.\operatorname{Im}\left[\delta \Pi_{\mathrm{pt}}^{p}\left(z \tilde{\omega}_{c}\right)\right]\right)-\langle\bar{q} q\rangle\left(m_{Q}\right)\left[1+\frac{2}{3} \frac{\alpha_{s}}{\pi}\left(1-3 \frac{\tilde{\omega}_{c}}{m_{Q}} \int_{0}^{\infty} \frac{\mathrm{d} z}{\left(1+z \tilde{\omega}_{c} / m_{Q}\right)^{n+2}}\right)\right] \\
& \left.+\frac{n(n+1)}{8} \frac{m_{0}^{2}\langle\bar{q} q\rangle}{m_{Q}^{2}}+\frac{\left\langle\alpha_{s} G^{\mu \nu} G_{\mu \nu}\right\rangle}{12 \pi m_{Q}}\right\} .
\end{aligned}
$$

\section{The heavy quark masses}

Before turning to the numerical analysis we want to discuss the determination of the heavy quark mass which is an input parameter of the sum rules given above. The result for the physical decay constant (30) is rather sensitive to the heavy quark mass value. Therefore $m_{Q}$ should be determined with a great accuracy in order to obtain a reasonable precision for $f_{P}$. The best accuracy of the bottom quark mass determination is achieved within the heavy quarkonium sum rules [2]. The corresponding expression for the pole mass is given by the ratio

$$
m_{b}=\left(\frac{\mathcal{M}_{n}^{\text {th }}}{\tilde{\mathcal{M}}_{n}^{\text {exp }}}\right)^{\frac{1}{2 n}}
$$

Here the dimensionful experimental moments

$$
\tilde{\mathcal{M}}_{n}^{\exp }=9 \int_{0}^{\infty} \mathrm{d} s \frac{R^{\exp }(s)}{s^{n+1}}
$$

are generated by the normalized cross section of $e^{+} e^{-}$annihilation $R^{\exp }(s)=\sigma\left(e^{+} e^{-} \rightarrow\right.$ hadrons $\left._{b \bar{b}}\right) / \sigma\left(e^{+} e^{-} \rightarrow \mu^{+} \mu^{-}\right)$. The dimensionless theoretical moments are defined as follows

$$
\mathcal{M}_{n}^{\text {th }}=12 \pi\left(4 m_{b}^{2}\right)^{n} \int_{0}^{\infty} \mathrm{d} s \frac{\operatorname{Im}\left[\Pi^{v}(s)\right]}{s^{n+1}}
$$

where the vector current correlator is defined through

$$
\left(q^{\mu} q^{\nu}-q^{2} g^{\mu \nu}\right) \Pi^{v}\left(q^{2}\right)=i \int \mathrm{d} x e^{i q x}\left\langle 0\left|T j^{\mu}(x) j^{\nu}(0)\right| 0\right\rangle
$$

with $j_{\mu}=\bar{b} \gamma_{\mu} b$. If $n$ is large enough the experimental moments are saturated by the $\Upsilon$ resonance contributions which is known with high precision. For large $n$ the dispersion integral in Eq. (36) is saturated by the region near threshold where the nonrelativistic expansion in the heavy quark velocity is applicable and the correlator can be systematically computed within the effective theory of nonrelativistic QCD (NRQCD) [26]. The complete result for the moments including the second order corrections in the strong coupling constant and heavy quark velocity is now available [22, 27, 28, 29.

It is widely believed that due to the renormalon contributions the absolute value of the heavy quark pole mass obtained through Eq. (34) is divergent [29, 30, 31]. As a

consequence the absolute value of the pole mass is plagued with an intrinsic uncertainty 
of order $\Lambda_{Q C D}$. On the other hand, $m_{b}$ is not an observable and has no immediate physical meaning. Therefore it can safely be removed from relations between physical observables. Using this philosophy we replace $m_{b}$ in the sum rules for $f_{B}$ by the fixed order expression of the right-hand side of Eq. (34). Equivalently, we determine the value to the pole mass according to Eq. (34) only in a given order of the perturbative expansion correlated to the order of the approximation for $f_{B}$. A detailed discussion of the sum rules and the corresponding numerical results can be found in [22]. In particular, we use the next-toleading order (NLO) result, $m_{b}=4.68 \mathrm{GeV}$, for the calculation of $f_{B}$ to order $\alpha_{s}$ and the next-to-next-to-leading order (NNLO) result, $m_{b}=4.79 \mathrm{GeV}$, for the $\mathcal{O}\left(\alpha_{s}^{2}\right)$ analysis of $f_{B}$. The accuracy of the numerical value for the fixed order approximation for $m_{b}$ is no longer restricted by $\Lambda_{Q C D}$ since it is not related to the divergence of the series in Eq. (34). It is mainly due to the dependence of the theoretical moments on the normalization scale of $\alpha_{s}$ and due to the dependence on $n$ which altogether amounts to $\pm 60 \mathrm{MeV}$ for the NNLO result [22, 27]. Note that the direct order-by-order matching of the results for $m_{b}$ and $f_{B}$ is not obvious due to different kinds of resummation adopted in the sum rules: the study of the $\Upsilon$-resonance sum rules requires the resummation of the singular Coulomb terms while the above analysis of the $B$-meson sum rules involves the resummation of the heavy quark mass logarithms. Our matching of the perturbative series is based on the fact that in a given approximation both the $\Upsilon$-resonance sum rules and the $B$-meson sum rules include the perturbative corrections of the same order in $\alpha_{s}$ to the threshold behaviour of the heavy-heavy and heavy-light quark current correlators, respectively. Performing the analysis in the described way we expect that the large perturbative corrections to $m_{b}$ cancel in the complete expression so that the final series for $f_{B}$ in terms of physical moments (35) is convergent. This approach turned out to be very efficient for the analysis of the bottom quark semileptonic decay width [21, 22]. We will show that the method works in the case of the sum rules for $f_{B}$ as well.

For a given value of the bottom quark mass the charm quark mass can be obtained from the HQET constraint of the form

$$
m_{b}-m_{c}+\mathcal{O}\left(1 / m_{b, c}\right)=M_{B}-M_{D},
$$

which results in $m_{c}=1.37 \mathrm{GeV}$ for the NNLO value of $m_{b}$. Due to the cancellation between the different terms of order $1 / m_{b, c}$ (see, for example, [32]) this numerical value is valid with $\mathcal{O}\left(1 / m_{b, c}\right)$ accuracy. The use of the relation (38) brings an additional uncertainty to $m_{c}$ so that the total uncertainty can be roughly estimated as $\pm 100 \mathrm{MeV}$.

\section{$6 \quad$ Numerical analysis}

In this section we present the numerical analysis of the sum rules. We adopt the same input values for the vacuum condensates as in [17:

$$
\begin{aligned}
\langle\bar{q} q\rangle(1 \mathrm{GeV}) & =-(225(25) \mathrm{MeV})^{3}, \\
\left\langle\alpha_{s} G^{\mu \nu} G_{\mu \nu}\right\rangle & =0.04(2) \mathrm{GeV}^{4}, \\
m_{0}^{2}(1 \mathrm{GeV}) & =0.8(2) \mathrm{GeV}^{2} .
\end{aligned}
$$


The strong coupling constant is evaluated with four active flavours using two-loop accuracy and $\Lambda^{(4)}=296 \mathrm{MeV}$. This value corresponds to $\alpha_{s}^{(5)}\left(m_{b}\right)=0.210$ obtained from $\alpha_{s}^{(5)}\left(M_{Z}\right)=0.1185$ using the four-loop renormalization group evolution [33]. For the meson masses we use $M_{B}=5.2793(7) \mathrm{GeV}$ and $M_{D}=1.8641(10) \mathrm{GeV}$, respectively [34].

\subsection{The decay constant within heavy quark effective theory}

Let us start with the analysis of the limit $m_{Q} \rightarrow \infty$. The general philosophy for the determination of the decay constant from Laplace sum rules is as follows [3]: one has to optimize the upper bound of the duality interval, $\tilde{\omega}_{c}$, in such a way that the value of $\tilde{f}_{P}^{r}$ as computed from Eq. (24) is stable against a variation of the Borel parameter $T$. The latter is varied in the range where both the hadronic and QCD representations of the correlator can be computed reliably. On the QCD side of the sum rules the restriction on $T$ is mainly due to the perturbative contribution because $T$ is an effective scale of $\alpha_{s}$ in Eq. (24). Taking into account the large value of the second order nonlogarithmic coefficient in Eq. (201) we conclude that $T$ cannot be chosen much less then $1.5 \mathrm{GeV}$ where $\alpha_{s}(T) / \pi \gtrsim 0.1$ to ensure the convergence of the perturbative series. Note that the power suppressed terms become dangerous at essentially lower $T$ and that the above restriction also provides the convergence of the OPE. On the opposite side of the sum rules the hadronic representation of the correlator is reliable only for $T \lesssim \tilde{\omega}_{c}$ which provides the exponential suppression of the contributions from higher resonances.

The logarithmic dependence of $\tilde{\Pi}_{\mathrm{pt}}(z T)$ on $T$ in Eq. (24) is quite important for the stability of the sum rules. Therefore it is crucial to use the HQET renormalization group to get control over the high order logarithmic contributions. The leading and next-toleading logarithms of $T$ can be summed up by setting $\mu=T$ in the factor $X(\mu)$ and in the correlator $\tilde{\Pi}_{\mathrm{pt}}(z T)$. We adopt this prescription in our analysis. However, the normalization scale of $\alpha_{s}$ in the $\mathcal{O}\left(\alpha_{s}^{2}\right)$ part of $\tilde{\Pi}_{\mathrm{pt}}(z T)$ is not fixed in our approximation and the corresponding $\mu$-dependence is not compensated by $X(\mu)$. We do not use $T$ as the normalization scale here when determining the optimal value of $\tilde{\omega}_{c}$ because the resulting spurious $T$-dependence leads to rather unstable sum rules. If the normalization scale of $\alpha_{s}$ in the $\mathcal{O}\left(\alpha_{s}^{2}\right)$ contribution is not correlated to $T$ the result has a rather weak dependence on $\mu$ when varying the latter in the same interval as $T$.

Adopting the central values of the input parameters and $\mu=2 \mathrm{GeV}$, we obtain for the universal HQET decay constant

$$
\tilde{f}_{P}^{r}=410(\mathrm{MeV})^{3 / 2},
$$

which constitutes an average for $2.05 \mathrm{GeV} \leq \tilde{\omega}_{c} \leq 2.10 \mathrm{GeV}$. For these values the highest stability is observed. This value should be compared with the result obtained using the order $\alpha_{s}$ expression of the correlator $\tilde{\Pi}_{\mathrm{pt}}(\tilde{\omega})$ which is $\tilde{f}_{P}^{r}=418 \mathrm{MeV}$ at the optimal value $\tilde{\omega}_{c}=2.4 \mathrm{GeV}$. One notices that the inclusion of the $\mathcal{O}\left(\alpha_{s}^{2}\right)$ contribution leads to a rather small variation of $\tilde{f}_{P}^{r}$ though the correction to the correlator itself is quite large (cf. Eq. (20)). This can be explained by a considerable compensation of the large corrections 


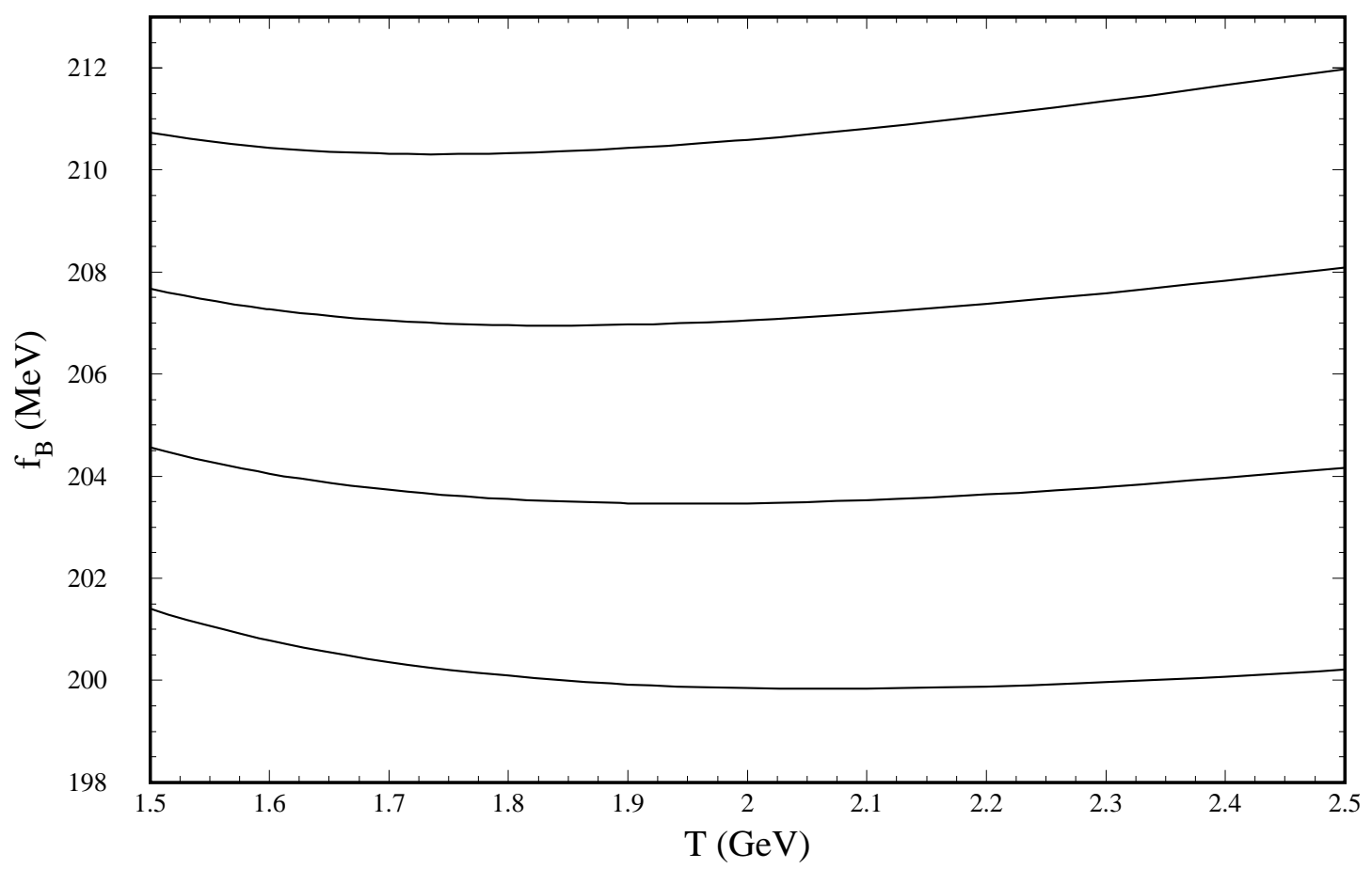

Figure 1: The $B$ meson decay constant $f_{B}$ as a function of the Borel parameter $T$ of the Laplace sum rules for different values of the threshold parameter $\tilde{\omega}_{c}$. From top to bottom the curves correspond to $\tilde{\omega}_{c}=2.35,2.3,2.25$ and $2.2 \mathrm{GeV}$.

to $\tilde{\Pi}_{\mathrm{pt}}(\tilde{\omega})$ and $m_{b}$ (which enters the analysis through $\Delta$ ) and the change of the optimal value $\tilde{\omega}_{c}$.

\section{2 $B$ meson decay constant}

Taking into account the mass suppressed contribution to the Laplace sum rules and performing the analysis along the line described in the previous section, we obtain for the $B$ meson decay constant

$$
f_{B}=206 \mathrm{MeV},
$$

which constitutes an average for $2.25 \mathrm{GeV} \leq \tilde{\omega}_{c} \leq 2.3 \mathrm{GeV}$. To illustrate the stability of the sum rules with respect to the variation of the parameter $T$ we plot in Fig. 1 f $f_{B}$ as a function of $T$ for various values of $\tilde{\omega}_{c}$. It can be seen that the curves for $\tilde{\omega}_{c} \approx 2.25 \mathrm{GeV}$ and $\tilde{\omega}_{c} \approx 2.3 \mathrm{GeV}$ provide the most stable results. Note that for these values of $\tilde{\omega}_{c}$ the function $f_{B}(T)$ has a weakly pronounced minimum around $T \approx 2 \mathrm{GeV}$. At the same time, the use of the two-loop $\mathcal{O}\left(\alpha_{s}\right)$ approximation of $\tilde{\Pi}_{\mathrm{pt}}(\tilde{\omega})$ along with the NLO value of $m_{b}$ gives $f_{B}=205 \mathrm{MeV}$ at the optimal value $\tilde{\omega}_{c}=2.75 \mathrm{GeV}$. As in the heavy quark limit we observe that due to the compensation of the corrections the $\mathcal{O}\left(\alpha_{s}^{2}\right)$ result is practically the same as the $\mathcal{O}\left(\alpha_{s}\right)$ one. This fact is a strong argument in favour of our treatment of 
the bottom quark mass. The $\mathcal{O}\left(\alpha_{s}^{2}\right)$ mass suppressed corrections, which are included in Eq. (41), reduce the value of $f_{B}$ by approximately $5 \mathrm{MeV}$. Taking into account the fact that the nonperturbative part of the QCD contribution is saturated by the leading quark condensate, which gives about $10 \%$ of the total QCD contribution, we conclude that our result is stable with respect to all the types of corrections to the sum rules.

Note that no rigorous results concerning the high order behaviour of the perturbative series both for the $\Upsilon$-resonance and $B$-meson sum rules are available and the absence of sizable higher order perturbative corrections to the decay constant within our approach cannot be proven strictly even though it works well up to the $\mathcal{O}\left(\alpha_{s}^{2}\right)$ approximation. However, our approach provides the convergence also in higher orders if the divergence of the perturbative series for the $\Upsilon$-resonance and $B$-meson sum rules is related to the use of the pole mass and is thus dominated by the renormalon contribution. This is because we effectively remove the pole mass from the analysis of the decay constant in favour of the experimental moments of the $\Upsilon$-resonance spectral density and operate with the relation between physical observables which is free from the corresponding renormalon ambiguity.

Let us next discuss the uncertainty of the result in Eq. (41). The error in $m_{b}$ of $\pm 60 \mathrm{MeV}$ [21, 22] results in an uncertainty of $\pm 12 \mathrm{MeV}$ in $f_{B}$. The variation of the input value of $\alpha_{s}\left(M_{Z}\right)$ within the experimental error bars $0.1185 \pm 0.0020$ [34] leads to the uncertainty interval $201 \mathrm{MeV}<f_{B}<213 \mathrm{MeV}$. On the other hand, the result is not sensitive to the normalization point of $\alpha_{s}$ in the $\mathcal{O}\left(\alpha_{s}^{2}\right)$ contribution and to the nonlogarithmic three-loop coefficient in Eq. (20). Note, that the change of the parameters requires every time a new optimization of $\tilde{\omega}_{c}$.

Another source of errors is the intrinsic uncertainty of the method due to the approximation of the hadronic contribution to the dispersion integral (8). A rough estimate of this uncertainty is obtained by the variation of the upper bound of the duality interval around its optimal value. The variation of $\tilde{\omega}_{c}$ by $\pm 100 \mathrm{MeV}$ leads to $\pm 7 \mathrm{MeV}$ variation of $f_{B}$ which can be read off Fig. 1. A larger deviation from the optimal value leads to the essentially unstable sum rules. If we add the errors induced by the uncertainties in $m_{b}$, $\alpha_{s}$ and $\tilde{\omega}_{c}$ discussed so far in quadrature we obtain $f_{B}=206 \pm 16 \mathrm{MeV}$.

A more advanced way to estimate the intrinsic uncertainty of the sum-rule approach is to change the weight function in the dispersion integral and redo the analysis using the sum rules which operate with Hilbert moments of the correlator (10) instead of its Borel transform (9). The range of $n$ relevant for reliable predictions of Hilbert sum rules is, in fact, quite restricted. The contribution of the mixed condensate grows rapidly with $n$. Thus, requiring the convergence of the OPE sets an upper limit on $n$. For the bottom quark it is $n \approx 12$ where the contribution of the mixed condensate is approximately $2 / 3$ of the leading quark one. At the same time, to avoid strong dependence of the result on $\tilde{\omega}_{c}$, one should use $n>m_{b} / \tilde{\omega}_{c} \approx 3$. The optimal value of $\tilde{\omega}_{c}$ can be found by minimizing the dependence of the result on $n$ in the above interval which results to $\tilde{\omega}_{c} \approx 2.2 \mathrm{GeV}$. For this value the decay constant stays within the interval $192 \mathrm{MeV} \lesssim f_{B} \lesssim 195 \mathrm{MeV}$ as $n$ varies from 4 to 12 as can be seen in Fig. 2 where $f_{B}$ is plotted as a function of $n$. This result for $f_{B}$ is in good agreement with the value obtained from the Laplace sum rules.

Note, that in addition to the stability requirement there is a strong consistency check 


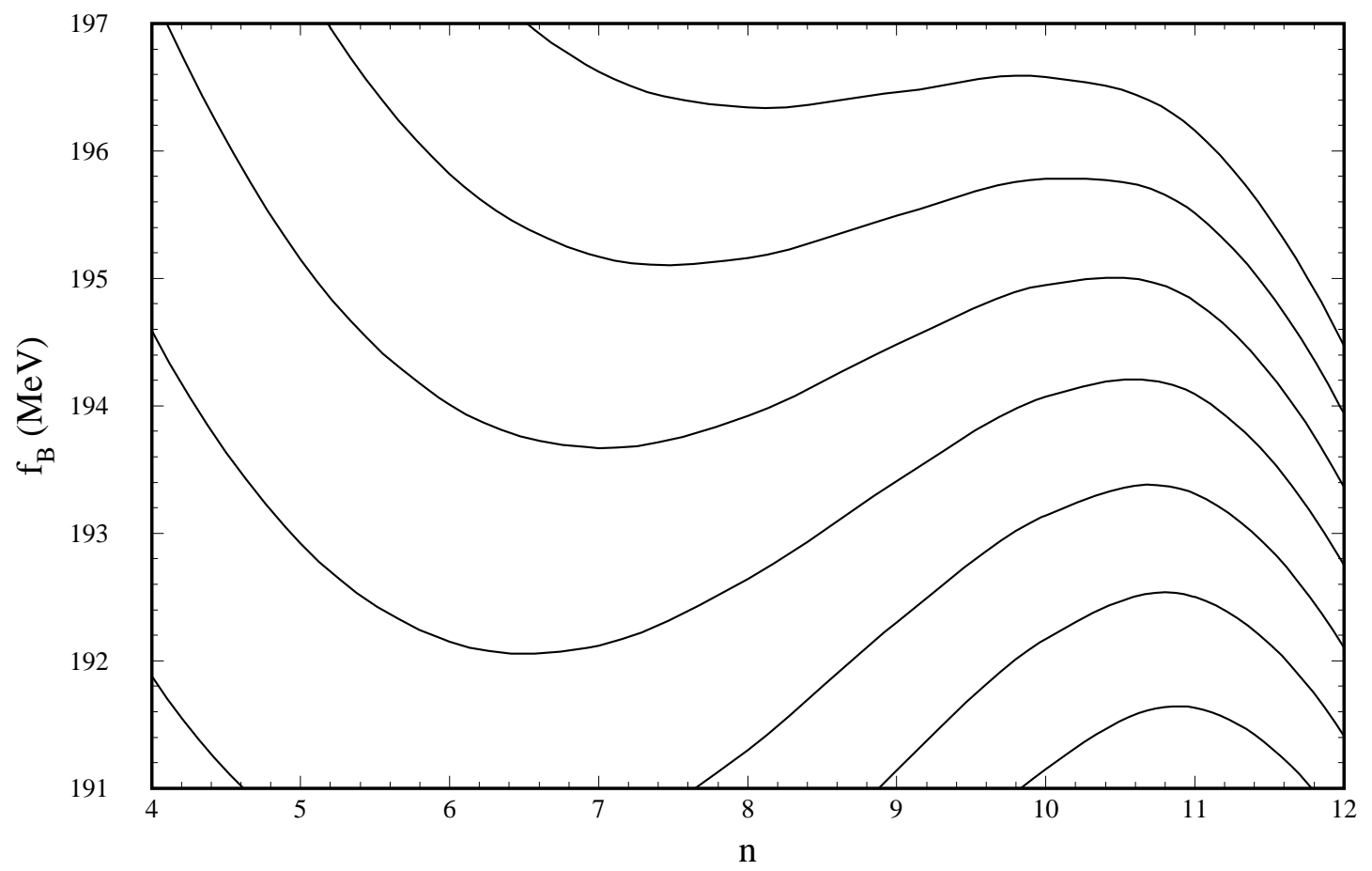

Figure 2: The $B$ meson decay constant $f_{B}$ as a function of the moment number $n$ of the Hilbert sum rules for different values of the threshold parameter $\tilde{\omega}_{c}$. From top to bottom the curves correspond to $\tilde{\omega}_{c}=2.35,2.3,2.25,2.2,2.15,2.1$ and $2.05 \mathrm{GeV}$. The value $\mu=2 \mathrm{GeV}$ has been adopted.

on the value of $\tilde{\omega}_{c}$. The decay constant drops out in the ratio of two moments which leads to a relation between $M_{B}, m_{b}$ and $\tilde{\omega}_{c}$. Our analysis is consistent if the physical value of the meson mass is reproduced from this relation for some value of the bottom quark mass in the interval given by the sum rules for the $\Upsilon$ resonances. For the above value of $\tilde{\omega}_{c}$ this requirement is fulfilled for all $n$ in the allowed interval. In fact, the ratio of the $6^{\text {th }}$ and $7^{\text {th }}$ moment and the ratio of the $10^{\text {th }}$ and $11^{\text {th }}$ moment imply exactly the central value $m_{b}=4.79$. Note that for these moments $f_{B}$ has a local extremum as a function of $n$. In other words, if the $B$-meson sum rules in the three-loop approximation are used to determine $m_{b}$, the result is in perfect agreement with the NNLO value of the bottom quark mass from the $\Upsilon$-resonances sum rules.

We would like to emphasize that the dependence of $f_{B}$ on $m_{b}$ is completely different for the Laplace and Hilbert sum rules. Thus, by comparing the results of the sum rules one can also estimate the error due to the uncertainty in $m_{b}$. Furthermore, the prescription how the parameter $\tilde{\omega}_{c}$ is determined and the structure of the condensate contributions are also quite different. The fact that both approaches give close results reflects the small intrinsic uncertainty of the sum-rule method in this particular case and furthermore strongly supports our treatment of $m_{b}$. Thus the total error originating from the uncertainty in $m_{b}$ and the approximation of the hadronic spectrum can be estimated 
as $\pm 15 \mathrm{MeV}$. The remaining error is mainly due to the uncertainty in the input values of $\alpha_{s}\left(M_{Z}\right)$. Consequently, as a conservative estimate of the uncertainty of our result for $f_{B}$ we quote $\pm 20 \mathrm{MeV}$. Because of the stronger dependence of the Hilbert sum rules on $m_{b}$ we use them to estimate the error but take the central value for our final result for $f_{B}$ from the Laplace sum rules.

\section{3 $D$ meson decay constant}

Since $m_{c}$ is not large in comparison to the scale $\bar{\Lambda}$ the expansion in $1 / m_{c}$ can not provide us with the same accuracy as we have for the bottom quark. By the same reason no resummation of the charm quark mass logarithms is necessary and we can just use the three-loop result for the correlator up to $\mathcal{O}\left(\alpha_{s}^{2}\right)$ in the full theory [19, 20]. The use of the Laplace sum rules then leads to

$$
f_{D}=195 \mathrm{MeV},
$$

for the optimal value $\tilde{\omega}_{c}=2.35 \mathrm{GeV}$. The Hilbert sum rules are not reliable in this case because of the strong dependence on $m_{c}$ which is known with much less relative accuracy than $m_{b}$. Furthermore, the Hilbert sum rules suffer from large contribution from the higher dimension condensates. The variation of the input parameters basically leads to similar variations of $f_{D}$ and $f_{B}$. Due to the weaker sensitivity of $f_{D}$ to the charm quark mass the additional uncertainty in $m_{c}$ does not lead to a larger error in $f_{D}$. Thus, assuming the same intrinsic uncertainty of the sum rules we obtain the same error bars for the extracted value of $f_{D}$.

\section{Conclusions}

To summarize, we have computed the $B$ and $D$ meson decay constants within the QCD sum rules approach. Our final results read

$$
\begin{aligned}
& f_{B}=206 \pm 20 \mathrm{MeV}, \\
& f_{D}=195 \pm 20 \mathrm{MeV} .
\end{aligned}
$$

For the analysis we used the tree-loop result for the heavy-light current correlator. The large logarithms of the bottom quark mass have been taken into account by means of the HQET renormalization group. The bottom quark mass which essentially limits the accuracy of the sum rules for $f_{B}$ has been extracted from the $\Upsilon$-resonance sum rules up to NNLO. In the case of the $B$ meson the analysis has been performed by employing Laplace and Hilbert sum rules. They have quite a different structure especially as regards the dependence on the bottom quark mass. The fact that the results obtained with these two approaches are in a good agreement gives us confidence in the reliability of the sumrule method applied to the calculation of the decay constants. The result also turned out to be quite stable with respect to inclusion of the perturbative corrections in $\alpha_{s}$ and $1 / m_{b}$ 
and the nonperturbative corrections due to the vacuum condensate contributions. This allowed us to reduce the uncertainty of the extracted value of $f_{B}$ and $f_{D}$. The obtained values of both the $B$ and $D$ meson decay constants are consistent with the existing sumrule results (cf. Eqs. (2) and (41)). However, the accuracy of our result is increased in comparison to the previous estimates. The values in Eqs. (43) and (44) are in impressive agreement with the results obtained in lattice calculations (cf. Eqs. (11) and (3)). Probably no further improvement of the accuracy is possible within the standard QCD sum-rule framework due to the intrinsic uncertainty of the method.

Our final comment concerns the current experimental status. A measurement is only available for the $D_{s}$ meson decay constant where the most recent result reads [35]

$$
f_{D_{s}}^{\text {exp }}=286 \pm 44(\text { stat }) \pm 41 \text { (syst) } \mathrm{MeV} .
$$

Converting the number in Eq. (44) with the help of the lattice result $f_{D_{s}} / f_{D}=1.18$ [18], which agrees with the values given in [17], we obtain $f_{D_{s}}=230 \mathrm{MeV}$. This is in reasonable agreement with the experimental value of Eq. (45).

\section{Acknowledgements}

We would like to thank K.G. Chetyrkin for the motivation to perfrom the calculations presented in this paper and A. Ali for useful comments and discussions. This work was supported in part by the Deutsche Forschungsgemeinschaft through Grant No. KN 365/1-1, by the Bundesministerium für Bildung und Forschung through Grant No. 05 HT9GUA 3, and by the European Commission through the Research Training Network Quantum Chromodynamics and the Deep Structure of Elementary Particles under Contract No. ERBFMRX-CT98-0194.

\section{References}

[1] T. M. Aliev and V. L. Eletsky, Sov. J. Nucl. Phys. 38 (1983) 936 [Yad. Fiz. 38 (1983) 1537].

[2] V. A. Novikov et al., Phys. Rev. Lett. 38 (1977) 626, (E) ibid. 38 (1977) 791; Phys. Rep. C 41 (1978) 1.

[3] M. A. Shifman, A. I. Vainshtein, and V. I. Zakharov, Nucl. Phys. B 147 (1979) 385; Nucl. Phys. B 147 (1979) 448.

[4] D. J. Broadhurst, Phys. Lett. B 101 (1981) 423.

[5] C. A. Dominguez and N. Paver, Phys. Lett. B 197 (1987) 423, [Erratum-ibid. B 199 (1987) 596].

[6] S. Narison, Phys. Lett. B 198 (1987) 104; Nucl. Phys. Proc. Suppl. 74 (1999) 304. 
[7] L. J. Reinders, Phys. Rev. D 38 (1988) 947.

[8] P. Colangelo, G. Nardulli, A. A. Ovchinnikov, and N. Paver, Phys. Lett. B 269 (1991) 201.

[9] C. A. Dominguez, in Proceedings of the Third Workshop on the Tau-Charm Factory, Marbella, Spain, 1-6 June (1993), Ed. J. Kirkby and R. Kirkby, Editions Frontiéres, p. 357.

[10] D. J. Broadhurst and A. G. Grozin, Phys. Lett. B 274 (1992) 421.

[11] E. Bagan, P. Ball, V. M. Braun, and H. G. Dosch, Phys. Lett. B 278 (1992) 457.

[12] M. Neubert, Phys. Rev. D 45 (1992) 2451.

[13] E. Eichten, B. Hill, Phys. Lett. B 234 (1990) 511;

H. Georgi, Phys. Lett. B 240 (1990) 447.

[14] M. Neubert, Phys. Rept. 245 (1994) 259.

[15] C. Bernard, Nucl. Phys. Proc. Suppl. 94 (2001) 159.

[16] A. Ali Khan et al. [CP-PACS Collaboration], hep-lat/0103020.

[17] P. F. Harrison and H. R. Quinn [BABAR Collaboration], SLAC-R-0504 Papers from Workshop on Physics at an Asymmetric B Factory (BaBar Collaboration Meeting), Rome, Italy, 11-14 Nov 1996, Princeton, NJ, 17-20 Mar 1997, Orsay, France, 16-19 Jun 1997 and Pasadena, CA, 22-24 Sep 199\%.

[18] A. Ali Khan et al. [CP-PACS Collaboration], Phys. Rev. D 64, 034505 (2001).

[19] K. G. Chetyrkin and M. Steinhauser, Phys. Lett. B 502 (2001) 104.

[20] K. G. Chetyrkin and M. Steinhauser, Report Nos.: DESY 01-090, THEP 01/07, TTP01-14 and hep-ph/0108017, Eur. Phys. J. C (in press).

[21] A. A. Penin and A. A. Pivovarov, Phys. Lett. B 443 (1998) 264.

[22] A. A. Penin and A. A. Pivovarov, Nucl. Phys. B 549 (1999) 217.

[23] D. J. Broadhurst and A. G. Grozin, Phys. Rev. D 52 (1995) 4082.

[24] A. G. Grozin, Phys. Lett. B 445 (1998) 165.

[25] X. Ji and M. J. Musolf, Phys. Lett. B 257 (1991) 409;

D. J. Broadhurst and A. G. Grozin, Phys. Lett. B 267 (1991) 105;

V. Giménez, Nucl. Phys. B 375 (1992) 582.

[26] W. E. Caswell and G. E. Lepage, Phys. Lett. B 167 (1986) 437;

G. E. Lepage et al., Phys. Rev. D 46 (1992) 4052. 
[27] J. H. Kühn, A. A. Penin, and A. A. Pivovarov, Nucl. Phys. B 534 (1998) 356;

A. A. Penin and A. A. Pivovarov, Phys. Lett. B 435 (1998) 413.

[28] A. H. Hoang, Phys. Rev. D 59 (1999) 014039.

[29] K. Melnikov and A. Yelkhovsky, Phys. Rev. D 59 (1999) 114009.

[30] M. Beneke and A. Signer, Phys. Lett. B 471 (1999) 233.

[31] A. H. Hoang, Phys. Rev. D 61 (2000) 034005.

[32] M. Neubert, "B Decays And the Heavy Quark Expansion", in the Second Edition of: Heavy Flavours, edited by A. J. Buras and M. Lindner (World Scientific, Singapore), hep-ph/9702375.

[33] K. G. Chetyrkin, J. H. Kühn and M. Steinhauser, Comput. Phys. Commun. 133 (2000) 43.

[34] D. E. Groom et al., Eur. Phys. J. C 15 (2000) 1.

[35] G. Abbiendi et al. [OPAL Collaboration], hep-ex/0103012. 